TRANSACTIONS OF THE

AMERICAN MATHEMATICAL SOCIETY

Volume 359, Number 6, June 2007, Pages 2721-2739

S 0002-9947(07)04071-8

Article electronically published on January 4, 2007

\title{
COMPACT OPERATORS AND NEST REPRESENTATIONS OF LIMIT ALGEBRAS
}

\author{
ELIAS KATSOULIS AND JUSTIN R. PETERS
}

\begin{abstract}
In this paper we study the nest representations $\rho: \mathcal{A} \longrightarrow \operatorname{Alg} \mathcal{N}$ of a strongly maximal TAF algebra $\mathcal{A}$, whose ranges contain non-zero compact operators. We introduce a particular class of such representations, the essential nest representations, and we show that their kernels coincide with the completely meet irreducible ideals. From this we deduce that there exist enough contractive nest representations, with non-zero compact operators in their range, to separate the points in $\mathcal{A}$. Using nest representation theory, we also give a coordinate-free description of the fundamental groupoid for strongly maximal TAF algebras.

For an arbitrary nest representation $\rho: \mathcal{A} \longrightarrow \operatorname{Alg} \mathcal{N}$, we show that the presence of non-zero compact operators in the range of $\rho$ implies that $\mathcal{N}$ is similar to a completely atomic nest. If, in addition, $\rho(\mathcal{A})$ is closed, then every compact operator in $\rho(\mathcal{A})$ can be approximated by sums of rank one operators $\rho(\mathcal{A})$. In the case of $\mathbb{N}$-ordered nest representations, we show that $\rho(\mathcal{A})$ contains finite rank operators iff $\operatorname{ker} \rho$ fails to be a prime ideal.
\end{abstract}

\section{INTRODUCTION}

One of the central themes in the representation theory of $\mathrm{C}^{*}$-algebras is the consideration of representations that contain non-zero compact operators in their ranges. For instance, it is a deep result of Glimm [11] that characterizes the type I $\mathrm{C}^{*}$-algebras as those $\mathrm{C}^{*}$-algebras whose irreducible representations always contain non-zero compact operators in their ranges. Other classes of $\mathrm{C}^{*}$-algebras relating to this line of thought are the $\mathrm{CCR}, \mathrm{GCR}$ and residually finite $\mathrm{C}^{*}$-algebras, to mention but a few.

Recently there has been an interest in the representation theory of non-selfadjoint algebras and in particular triangular subalgebras of $\mathrm{AF} \mathrm{C}^{*}$-algebras [3, 4, 5, 12, 22 . In the case of non-selfadjoint operator algebras, the irreducible representations are not always sufficient to capture the structure of the algebra, and therefore appropriate substitutes have been introduced. A representation $\rho$ of an operator algebra $\mathcal{A}$ is said to be a nest representation if the closed invariant subspaces of $\rho(\mathcal{A})$ are linearly ordered, i.e., form a nest. Lamoureux [16] coined the term $n$ primitive ideal for the kernel of a nest representation and showed [16, 18, that in various contexts of non-selfadjoint operator algebras the n-primitive ideals play a role analogous to the primitive ideals in $\mathrm{C}^{*}$-algebras. For instance, one can equip the set of n-primitive ideals with the hull-kernel topology, and for every (closed,

Received by the editors April 15, 2004 and, in revised form, March 27, 2005.

2000 Mathematics Subject Classification. Primary 47L80.

The first author's research was partially supported by a grant from ECU.

(C) 2007 American Mathematical Society Reverts to public domain 28 years from publication 
two-sided) ideal $\mathcal{J}$ in the algebra, $\mathcal{J}$ is the intersection of all n-primitive ideals containing $\mathcal{J}$.

In this paper we study nest representations whose ranges contain non-zero compact operators. The algebras under consideration are the strongly maximal TAF algebras, i.e., direct limits of sums of upper triangular matrices so that the corresponding embeddings are *-extendable and regular [25, 21, 26. Perhaps surprisingly, there is little in the literature concerning representations of strongly maximal TAF algebras with compact operators in the range. One reason may be that the ranges of the faithful representations of such algebras fail to contain any compact operators, provided that the enveloping $\mathrm{C}^{*}$-algebra is simple. This applies to all motivating examples of the theory, such as the standard, refinement and alternation limit algebras. The situation changes dramatically however if one allows the representation to have non-zero kernel. To begin with, every multiplicative linear form on the algebra generates a representation on a one-dimensional Hilbert space, and so its range consists of rank one operators. (We call these representations trivial, for obvious reasons.) More interestingly, the standard limit algebra has sufficiently many finite-dimensional representations to separate the points. (See [19] for a characterization of the residually finite TUHF algebras.) It is not obvious at all, however, that other algebras, such as the refinement or alternation limit algebras, admit non-trivial nest representations with non-zero compact operators in the range. It is this question that initially motivated the research in this paper. It turns out that this question is related to a different open problem in representation theory which we now describe.

An ideal $\mathcal{J}$ of a Banach algebra $\mathcal{A}$ is said to be meet irreducible iff it cannot be expressed as the intersection of a finite collection of ideals of $\mathcal{A}$ properly containing $\mathcal{J}$. The meet irreducible ideals are related to the n-primitive ideals, and this relationship was exploited in [16]. In [5] it was shown that for a strongly maximal TAF algebra every meet irreducible ideal is n-primitive. The converse question, whether every n-primitive ideal is meet irreducible, was left open in [5] and was answered affirmatively in 4 .

Another problem that was left open in [5] was the characterization of completely meet irreducible ideals using representation theory. An ideal $\mathcal{J}$ of a Banach algebra $\mathcal{A}$ is called completely meet irreducible if, for any collection of ideals $\mathcal{J}_{a}, a \in \mathbb{A}$, containing $\mathcal{J}$, the relation $\mathcal{J}=\bigcap_{a \in \mathbb{A}} \mathcal{J}_{a}$ implies $\mathcal{J}=\mathcal{J}_{a}$, for some $a \in \mathbb{A}$. It is easily seen that this definition is equivalent to the existence of a unique successor $\mathcal{J}^{+}$of $\mathcal{J}$ in the ideal lattice of $\mathcal{A}$. The completely meet irreducible ideals have played a fundamental role in the classification theory of strongly maximal TAF algebras 6, 7, 8. Motivated by this, and their study of nest representations, the five authors of [5] attempted a characterization of the completely meet irreducible ideals of a strongly maximal TAF algebra in terms of its nest representations (their Corollary 5.7). In [5. Example 5.8] it was realized that the order type of the nest alone is not sufficient for such a characterization, and therefore the problem was left open. The solution of this problem is contained in Theorem 2.3 and Corollary 2.5, It turns out that the missed point in [5] was the use of finite rank operators. Here we identify a special class of nest representations whose ranges contain compact operators, the essential nest representations (Definition 2.4), and we show that the completely meet irreducible ideals coincide with the kernels of the essential nest representations. 
A key element in the proof of Theorem 2.3 is our recent characterization of the meet irreducible ideals as the kernels of the nest representations 4 .

Utilizing the connection between compact operators and completely meet irreducible ideals, we subsequently show that every strongly maximal TAF algebra admits sufficiently many contractive representations, with compact operators in the range, to separate the points and actually capture the norm (Theorem 2.10). This answers affirmatively the question that stimulated this work.

Motivated by the significance of nest representations with compact operators in the range, we undertake a general study of such representations in the second section of the paper. A first result shows that if the range of a nest representation $\rho: \mathcal{A} \rightarrow \operatorname{Alg} \mathcal{N}$ of a strongly maximal TAF algebra $\mathcal{A}$ contains compact operators, then the nest $\mathcal{N}$ is similar to a completely atomic one. Having established that the nests involved in our considerations are completely atomic (up to a similarity), we subsequently consider the simplest possible case. In Theorem 3.3 we show that the range of an $\mathbb{N}$-ordered nest representation $\rho: \mathcal{A} \rightarrow \operatorname{Alg} \mathcal{N}$ contains finite rank operators if and only if ker $\rho$ fails to be a prime ideal. (It is worth mentioning that the proof of this result works for any complex algebra.) The second section concludes with a result which shows that if the range of a nest representation $\rho: \mathcal{A} \rightarrow \operatorname{Alg} \mathcal{N}$ is closed, then the compact operators in $\rho(\mathcal{A})$ form a closed ideal in $\operatorname{Alg} \mathcal{N}$. This allows us to make use of the theory in [10] and strengthen several of the results of this section, provided that $\rho(\mathcal{A})$ is norm closed. Finally, note that Example 2.6 shows that it is possible that the only non-zero compact operators in the range of a nest representation are rank one operators. Therefore we cannot expect stronger approximation results by utilizing strong operator topology. (Compare with Corollary [3.8.)

In the final section of the paper we use representation theory to give a coordinatefree description of the fundamental groupoid for a strongly maximal TAF algebra $\mathcal{A}=\lim \left(\mathcal{A}_{i}, \varphi_{i}\right)$, i.e., a description that does not depend on the ascending sequence of finite-dimensional subalgebras $\mathcal{A}_{i}, i \in \mathbb{N}$. Our approach is influenced from the definition of the projective space from Algebraic Geometry.

Remark 1.1. The results of Section 4 have influenced subsequent work on the classification problem for non-selfadjoint operator algebras. In [13], the first author and D. Kribs classify the quiver and free semigroupoid algebras of Muhly [20] and Kribs and Power [15] using a variant of the projective dual (Definition 4.2).

\section{Compact operators, REPRESENTATiOnS AND COMPLETELY MEET IRREDUCIBLE IDEALS}

In this section we characterize the completely meet irreducible ideals as kernels of nest representations of a special kind. Subsequently we show that for every strongly maximal TAF algebra there exist sufficiently many nest representations, containing finite rank operators in their ranges, to separate the points.

The following was also observed in [12] and will be repeatedly used in the sequel.

Lemma 2.1. Let $\rho: \mathcal{A} \rightarrow \operatorname{Alg} \mathcal{N}$ be a nest representation of a strongly maximal TAF algebra $\mathcal{A}=\lim \left(\mathcal{A}_{i}, \varphi_{i}\right)$ on a Hilbert space $\mathcal{H}$. Assume that $\rho$ is a

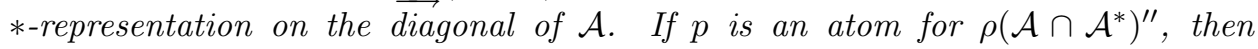
$\operatorname{dim} p(\mathcal{H})=1$. 
Proof. If $g \in p(\mathcal{H})$, then $[\rho(\mathcal{A}) g] \in \mathcal{N}$ and satisfies

$$
[\rho(\mathcal{A}) g]=[g] \oplus M_{g}
$$

for some closed subspace $M_{g} \subseteq p(\mathcal{H})^{\perp}$. Clearly any two such subspaces $[\rho(\mathcal{A}) g]$, $[p(\mathcal{A}) h]$ cannot be comparable unless $g=\lambda h, \lambda \in \mathbb{C}$, and the conclusion follows.

If $\mathcal{N}$ is a nest, then $0_{+}$denotes the minimal non-zero element of $\mathcal{N}$ with the understanding that if such an element does not exist, then $0_{+}=0$. Similarly, $I_{-}$ denotes the largest element of $\mathcal{N}$ not equaling $I$.

Lemma 2.2. Let $\mathcal{A}=\lim \left(\mathcal{A}_{i}, \varphi_{i}\right)$ be a strongly maximal TAF algebra and let $\rho: \mathcal{A} \rightarrow \operatorname{Alg} \mathcal{N}$ be a nest representation of $\mathcal{A}$ on a Hilbert space $\mathcal{H}$. Assume that $R \in \operatorname{Alg} \mathcal{N}$ is a non-zero rank one operator so that

$$
R \rho(\mathcal{A})=\rho(\mathcal{A}) R=\mathbb{C} R .
$$

Then there exist non-zero vectors $g, h \in \mathcal{H}$ so that $0_{+}=[g], I_{-}^{\perp}=[h]$ and $R=h \otimes g$.

Proof. Let $R=g \otimes h$ for suitable vectors $g, h \in \mathcal{H}$. Now $\rho(\mathcal{A}) R=\mathbb{C} R$ implies that $[\rho(\mathcal{A}) g]=[g]$, i.e., the subspace $[g]$ is invariant by $\rho(\mathcal{A})$. Since $\rho: \mathcal{A} \rightarrow \operatorname{Alg} \mathcal{N}$ is a nest representation, we obtain $[g] \in \mathcal{N}$. Therefore, $0_{+}=[g]$. A similar argument shows that $I_{-}^{\perp}=[h]$, and the conclusion follows.

Corollary 5.7 of [5] shows that for a certain class of nest representations whose kernels are completely meet irreducible ideals, 0 has an immediate sucessor in the nest, and $I$ has an immediate predecessor. In the following theorem we require merely that the nest representation be a star representation on the diagonal.

Theorem 2.3. Let $\mathcal{A}=\lim \left(\mathcal{A}_{i}, \varphi_{i}\right)$ be a strongly maximal TAF algebra and let $\rho: \mathcal{A} \rightarrow \operatorname{Alg} \mathcal{N}$ be a nest representation of $\mathcal{A}$ on a Hilbert space $\mathcal{H}$. If $\rho$ is a *-representation on the diagonal of $\mathcal{A}$, then the following are equivalent:

(i) $\operatorname{ker} \rho$ is a completely meet irreducible ideal.

(ii) There exist non-zero vectors $g, h \in \mathcal{H}$ so that $0_{+}=[g], I_{-}^{\perp}=[h]$ and $R=h \otimes g \in \rho(\mathcal{A})$.

Proof. Assume that $\mathcal{J}=\operatorname{ker} \rho$ is a completely meet irreducible ideal. Lemma 3.1 in 8 now implies that there exists a closed ideal $\mathcal{J}^{+}$containing $\mathcal{J}$ so that $\operatorname{dim}\left(\mathcal{J}^{+} / \mathcal{J}\right)=1$. Since closed ideals of limit algebras are inductive, there exists a matrix unit $e \in \mathcal{A}_{n}, n \in \mathbb{N}$, so that $e \in \mathcal{J}^{+} \backslash \mathcal{J}$. Furthermore, all but one of the subordinates of $e$ in $\mathcal{A}_{i}, i \geq n$, are mapped by $\rho$ to zero, or otherwise $\operatorname{dim}\left(\mathcal{J}^{+} / \mathcal{J}\right) \neq 1$. Therefore we produce decreasing sequences $\left\{p_{i}\right\}_{i \geq n},\left\{q_{i}\right\}_{i \geq n}$ of diagonal matrix units, $p_{i}, q_{i} \in \mathcal{A}_{i}, i \geq n$, so that $p_{i} e q_{i}$ is a subordinate of $e$ and

$$
\rho\left(p_{i} e q_{i}\right)=\rho(e) .
$$

Note that the sequences $\left\{\rho\left(p_{i}\right)\right\}_{i \geq n},\left\{\rho\left(q_{i}\right)\right\}_{i \geq n}$ are decreasing sequences of selfadjoint projections and therefore converge strongly to projections $p$ and $q$, respectively.

We claim that $p$ and $q$ are one-dimensional projections commuting with $\mathcal{N}$.

First note that (10) shows that $p \rho(e) q=\rho(e)$, and so both $p$ and $q$ are non-zero projections. Now for a fixed $i \in \mathbb{N}$,

$$
p_{i+k} \mathcal{A}_{i} p_{i+k}=p_{i+k} p_{i} \mathcal{A}_{i} p_{i} p_{i+k}=\mathbb{C} p_{i+k}
$$

for all $k \in \mathbb{N}$. Hence, $p \rho\left(\mathcal{A}_{i}\right) p=\mathbb{C} p$ and so $p \rho(\mathcal{A}) p=\mathbb{C} p$. In particular, $p$ is an atom for $\rho\left(\mathcal{A} \cap \mathcal{A}^{*}\right)^{\prime \prime}$, and so Lemma 2.1 shows that $p$ is a rank one projection commuting with $\mathcal{N}$. A similar argument proves the claim for $q$. 
Since $p \rho(e) q=\rho(e)$ the above claim shows that $\rho(e)$ is a rank one operator and so there exist vectors $g, h \in \mathcal{H}$ so that $\rho(e)=h \otimes g$. Furthermore note that given any matrix unit $a \in \mathcal{A}$, we have $a e \in \mathcal{J}^{+}$and so either $a e=\lambda e+f, \lambda \in \mathbb{C}, f \in \mathcal{J}$, or ae $\in \mathcal{J}$ (and $f=0$ ). In any case, $\rho(a) \rho(e)=\lambda \rho(e)$ for some $\lambda \in \mathbb{C}$. Hence, $\rho(\mathcal{A}) \rho(e)=\mathbb{C} \rho(e)$ and similarly $\rho(e) \rho(\mathcal{A})=\mathbb{C} \rho(e)$, i.e., $\rho(e)$ satisfies the condition of Lemma 2.2 and the conclusion follows.

We now show that (ii) implies (i). A moment's reflection shows that $\mathbb{C} R$ is an ideal in $\operatorname{Alg} \mathcal{N}$ and therefore in $\rho(\mathcal{A})$. Hence, $\hat{\mathcal{J}}=\rho^{-1}(\mathbb{C} R)$ is a norm closed ideal of $\mathcal{A}$ containing $\operatorname{ker} \rho$. Since $\operatorname{dim}(\hat{\mathcal{J}} / \operatorname{ker} \rho)=1$, there exists $a \in \mathcal{A}$ so that

$$
\hat{\mathcal{J}}=\mathbb{C} a+\operatorname{ker} \rho .
$$

Let $\mathcal{I}$ be any ideal properly containing $\operatorname{ker} \rho$. Then

$$
\operatorname{ker} \rho \subseteq \mathcal{I} \cap \hat{\mathcal{J}} \subseteq \hat{\mathcal{J}}
$$

with one of the above two containments being an equality because of (2). However, ker $\rho$ is the kernel of a nest representation and so Theorem 2.4 in [4 implies that $\operatorname{ker} \rho$ is a meet irreducible ideal. Hence, $\mathcal{I} \cap \hat{\mathcal{J}}=\hat{\mathcal{J}}$ and so $a \in \mathcal{I}$. This shows that any closed ideal containing $\operatorname{ker} \rho$ also contains $a$ and so the intersection of all such ideals properly contains $\operatorname{ker} \rho$. Conclusion: $\operatorname{ker} \rho$ is a completely meet irreducible ideal.

The statement of Theorem 2.3 suggests the following definition.

Definition 2.4. A nest representation $\rho: \mathcal{A} \rightarrow \operatorname{Alg} \mathcal{N}$ of an operator algebra $\mathcal{A}$ is said to be essential if the following conditions are satisfied:

(i) $\operatorname{dim} 0_{+}=\operatorname{dim} I_{-}^{\perp}=1$.

(ii) The rank one operator from $I_{-}^{\perp}$ into $0_{+}$belongs to $\rho(\mathcal{A})$.

We have arrived at the desired characterization of completely meet irreducible ideals.

Corollary 2.5. A closed ideal of a strongly maximal TAF algebra is completely meet irreducible ideal if and only if it is the kernel of an essential nest representation.

Proof. Observe that the proof of (ii) implies (i) in Theorem 2.3 is applicable to any essential nest representation. (The requirement that $\rho$ is a $*$-homomorphism on the diagonal is used in the proof of the other direction.) Therefore, the kernels of the essential nest representations are completely meet irreducible ideals. Conversely, any meet irreducible ideal $\mathcal{J}$ of a strongly maximal TAF algebra $\mathcal{A}$ is the kernel of a nest representation $\rho$, which is a $*$-representation on the diagonal [5. If $\mathcal{J}$ is completely meet irreducible, then the above theorem shows that $\rho$ is an essential nest representation and the conclusion follows.

In [5. Example 5.8] it is shown that the above result fails if one does not require the range of the essential nest representation to contain the designated rank one operator. On the other hand, it is easy to see that for the refinement algebras, the order type of the nest alone implies the existence of the rank one operator in Definition 2.4 It would be interesting to know which are precisely the algebras which satisfy that property.

The following examples show that the finite rank operators contained in the range of an essential nest representation may not be sufficient to describe the range 
of the representation in any reasonable topology. To give the examples, we require the theory of $\mathrm{C}^{*}$-envelopes as developed in 3.

Let $\mathfrak{A}=\lim \left(\mathfrak{A}_{i}, \varphi_{i}\right)$ be the enveloping $\mathrm{C}^{*}$-algebra for a TAF algebra $\mathcal{A}=$ $\underline{\lim }\left(\mathcal{A}_{i}, \varphi_{i}\right)$ and let $\mathcal{J} \subseteq \mathcal{A}$ be a closed ideal; let $\mathcal{J}_{i}:=\mathcal{J} \cap \mathcal{A}_{i}$. For each $i \geq 1, \mathcal{S}_{i}$

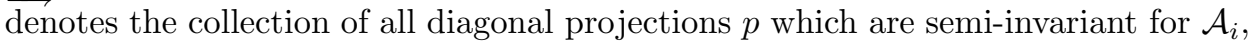
are supported on a single summand of $\mathfrak{A}_{i}$ and satisfy $\left(p \mathcal{A}_{i} p\right) \cap \mathcal{J}=\{0\}$. We form finite dimensional $\mathrm{C}^{*}$-algebras

$$
\mathfrak{B}_{i}:=\sum_{p \in S_{i}}^{\oplus} \mathcal{B}(\operatorname{Ran} p),
$$

where $\mathcal{B}(\operatorname{Ran} p)$ denotes the bounded operators on $\operatorname{Ran} p$; of course, $\mathcal{B}(\operatorname{Ran} p)$ is isomorphic to $\mathfrak{M}_{\text {rank } p}$. Let $\sigma_{i}$ be the map from $\mathfrak{A}_{i}$ into $\mathfrak{B}_{i}$ given by $\sigma_{i}(a)=$ $\left.\sum_{p \in S_{i}}^{\oplus} p a p\right|_{\operatorname{Ran} p}$. The map $\left.\sigma_{i}\right|_{\mathcal{A}_{i}}$ factors as $\rho_{i} q_{i}$, where $q_{i}$ is the quotient map of $\mathcal{A}_{i}$ onto $\mathcal{A}_{i} / \mathcal{J}_{i}$ and $\rho_{i}$ is a completely isometric homomorphism of $\mathcal{A}_{i} / \mathcal{J}_{i}$ into $\mathfrak{B}_{i}$. Note that $\mathfrak{B}_{i}$ equals the $\mathrm{C}^{*}$-algebra generated by $\rho_{i}\left(\mathcal{A}_{i} / \mathcal{J}_{i}\right)$.

We then consider unital embeddings $\pi_{i}$ of $\mathfrak{B}_{i}$ into $\mathfrak{B}_{i+1}$ defined as follows. For each $q \in \mathcal{S}_{i+1}$ we choose projections $p \in \mathcal{S}_{i}$ which maximally embed into $q$ under the action of $\varphi_{i}$. This way, we determine multiplicity one embeddings of $\mathcal{B}(\operatorname{Ran} p)$ into $\mathcal{B}(\operatorname{Ran} q)$. Taking into account all such possible embeddings, we determine the embedding $\pi_{i}$ of $\mathfrak{B}_{i}$ into $\mathfrak{B}_{i+1}$.

Finally we form the subsystem of the directed limit $\mathfrak{B}=\lim _{\longrightarrow}\left(\mathfrak{B}_{i}, \pi_{i}\right)$ corresponding to all summands which are never mapped into a summand $\mathcal{B}(\operatorname{Ran} p)$, where $p$ is a maximal element of some $\mathcal{S}_{i}$. Evidently this system is directed upwards. It is also hereditary in the sense that if every image of a summand lies in one of the selected blocks, then it clearly does not map into a maximal summand and thus already lies in our system. By [2, Theorem III.4.2], this system determines an ideal $\mathfrak{I}$ of $\mathfrak{B}$. The quotient $\mathfrak{B}^{\prime}=\mathfrak{B} / \mathfrak{I}$ is the AF algebra corresponding to the remaining summands and the remaining embeddings; it can be expressed as a direct limit $\mathfrak{B}^{\prime}=\underline{\lim }\left(\mathfrak{B}_{i}^{\prime}, \pi_{i}^{\prime}\right)$, with the understanding that $\mathfrak{B}_{i}^{\prime}=\bigoplus_{j} \mathfrak{B}_{i j}$ for these remaining summands $\mathfrak{B}_{i j}$ of $\mathfrak{B}_{i}$. It can be seen that the quotient map is isometric on $A / \mathcal{J}$ and that $\mathfrak{B}^{\prime}$ is the $\mathrm{C}^{*}$-envelope of $A / \mathcal{J}$.

Example 2.6. There exists an infinite-dimensional essential nest representation $\rho$ of a strongly maximal TAF algebra $\mathcal{A}$ so that the only compact operators in $\rho(\mathcal{A})$ are rank one operators.

Let $\mathcal{A}$ be the $2^{\infty}$-refinement algebra and let $\mathcal{J}$ be the ideal of $\mathcal{A}$ determined by the sequence

$$
e_{1,2}^{(1)}, e_{1,3}^{(2)}, e_{1,5}^{(3)}, e_{1,9}^{(4)}, \ldots
$$

of subordinates of the matrix unit $e_{1,2}^{(1)} \in \mathcal{A}_{1}$. Therefore, the ideal $\mathcal{A} \cap \mathcal{J}_{i}$ is generated by all the matrix units in $\mathcal{A}_{i}$ except from the ones in the "wedge" whose top right corner is $e_{1,2^{i-1}+1}^{(i)}$. Following the construction of the $\mathrm{C}^{*}$-envelope described earlier, the $\mathrm{C}^{*}$-envelope $\mathfrak{B}^{\prime}=\lim _{\longrightarrow}\left(\mathfrak{B}_{i}^{\prime}, \pi_{i}^{\prime}\right)$ of $\mathcal{A} / \mathcal{J}$ is given by the inductive limit

$$
M_{2} \oplus M_{1} \longrightarrow M_{3} \oplus M_{2} \longrightarrow M_{5} \oplus M_{4} \longrightarrow \cdots,
$$


where $M_{2^{i}}$ embeds in $M_{2^{i+1}}$ with the refinement embedding and $M_{2^{i}+1} \oplus M_{2^{i}}$ embeds in $M_{2^{i+1}+1}$ with the embedding that sends

$$
\left(\begin{array}{cccc}
a_{1,1} & a_{1,2} & \ldots & a_{1, k+1} \\
a_{2,1} & a_{2,2} & \ldots & a_{2, k+1} \\
\vdots & \vdots & \ddots & \vdots \\
a_{k+1,1} & a_{k+1,2} & \cdots & a_{k+1, k+1}
\end{array}\right) \oplus\left(\begin{array}{cccc}
b_{1,1} & b_{1,2} & \ldots & b_{1, k} \\
b_{2,1} & b_{2,2} & \ldots & b_{2, k} \\
\vdots & \vdots & \ddots & \vdots \\
b_{k, 1} & b_{k, 2} & \cdots & b_{k, k}
\end{array}\right)
$$

to the matrix

$$
\left(\begin{array}{cccccccc}
a_{1,1} & 0 & a_{1,2} & 0 & \ldots & a_{1, k} & 0 & a_{1, k+1} \\
0 & b_{1,1} & 0 & b_{1,2} & \ldots & 0 & b_{1, k} & 0 \\
a_{2,1} & 0 & a_{2,2} & 0 & \ldots & a_{2, k} & 0 & a_{2, k+1} \\
0 & b_{2,1} & 0 & b_{2,2} & \ldots & 0 & b_{2, k} & 0 \\
\vdots & \vdots & \vdots & \vdots & \ddots & \vdots & \vdots & \vdots \\
a_{k, 1} & 0 & a_{k, 2} & 0 & \ldots & a_{k, k} & 0 & a_{k, k+1} \\
0 & b_{k, 1} & 0 & b_{k, 2} & \ldots & 0 & b_{k, k} & 0 \\
a_{k+1,1} & 0 & a_{k+1,2} & 0 & \ldots & a_{k+1, k} & 0 & a_{k+1, k+1}
\end{array}\right) .
$$

We therefore have a commutative diagram

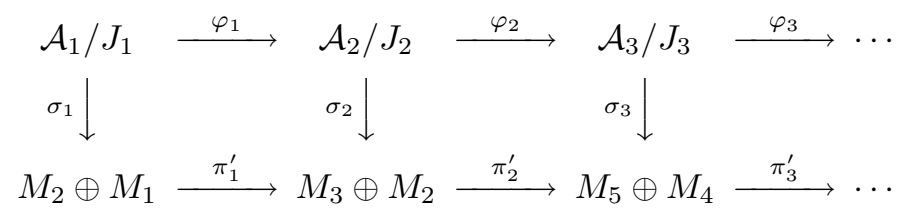

where the vertical maps $\sigma_{i}$ are defined as follows. Given $a \in \mathcal{A}_{i}$,

$$
\sigma_{i}(a+\mathcal{J})=\hat{a} \oplus \tilde{a}
$$

where $\hat{a}$ is the "wedge" of $a$ determined by $a+\mathcal{J}_{i}$ and $\tilde{a}$ results from $\hat{a}$ when its last row and column are removed.

Consider the representation $\tau: \mathfrak{B}^{\prime} \rightarrow \mathcal{B}(\mathcal{H})$ of Theorem 2.4 in [4. This is a faithful irreducible representation of $\mathrm{C}_{\text {env }}^{*}(\mathcal{A} / \mathcal{J})$ because $\mathcal{J}$ is meet irreducible. It also maps $\mathcal{A} / \mathcal{J}$ densely in a nest algebra $\operatorname{Alg} \mathcal{N}$. Let $\rho=\tau \circ \pi$, where $\pi: \mathcal{A} \rightarrow \mathcal{A} / \mathcal{J}$ is the quotient map.

Since $\mathcal{J}$ is a completely meet irreducible ideal, there exist non-zero vectors $g, h \in$ $\mathcal{H}$ so that $0_{+}=[h], I_{-}^{\perp}=[g]$. We claim that the only compact operators in $\rho(\mathcal{A})=\tau(\mathcal{A} / \mathcal{J})$ are of the form $g \otimes h^{\prime}, h^{\prime} \in \mathcal{H}$.

We first identify the rank one operators in $\tau\left(\bigcup_{i \in \mathbb{N}} \mathfrak{B}_{i}^{\prime}\right)$. Let $e \in \bigcup_{i \in \mathbb{N}} \mathfrak{B}_{i}^{\prime}$. Since $\tau$ is an irreducible representation, $\tau(e)$ has rank one iff

$$
e \mathfrak{B}^{\prime} e=\mathbb{C} e,
$$

i.e., $e$ has only one subordinate in any $\mathfrak{B}_{i}^{\prime}$. Therefore, $e \in M_{2^{i+1}+1}$, for some $i \in \mathbb{N}$. If in addition $e$ belongs to $\mathcal{A} / \mathcal{J}$, then (3) shows that all columns of $e$ are 0 except the last one. For such a $e, \tau(e)$ has the claimed form.

Finally, we look at $\mathcal{A}$ and consider $v \in \bigcup_{i \in \mathbb{N}} \mathcal{A}_{i}$ so that $\rho(v)$ is a rank one operator. Then $\pi(v)$ is mapped by $\tau$ to a rank one operator and therefore $\rho(v)=\tau(\pi(v))$ has the claimed form. By Corollary [3.8, sums of these operators approximate all compacts in $\rho(\mathcal{A})$, and the conclusion follows.

With some additional work, one can obtain the following. 
Example 2.7. There exists an infinite-dimensional essential nest representation $\rho$ of a strongly maximal TAF algebra $\mathcal{A}$ so that the only compact operators in $\rho(\mathcal{A})$ are the scalar multiples of the rank one operator $R$ appearing in Theorem 2.3 .

Let $\mathcal{A}$ be the $2^{\infty}$-refinement algebra, and this time let $\mathcal{J}$ be the ideal of $\mathcal{A}$ determined by the sequence

$$
e_{1,2}^{(1)}, e_{2,4}^{(2)}, e_{3,7}^{(3)}, e_{6,14}^{(4)}, \ldots
$$

of subordinates of the matrix unit $e_{1,2}^{(1)} \in \mathcal{A}_{1}$.

We now show that for a strongly maximal TAF algebra $\mathcal{A}$, the nest representations whose ranges contain finite rank operators separate the points. First we need a lemma.

Lemma 2.8. Let $\mathcal{A}$ be a strongly maximal TAF algebra and let $e \in \mathcal{A}_{i}, i \in \mathbb{N}$, be a matrix unit. Then there exists a completely meet irreducible ideal $\mathcal{J} \subseteq \mathcal{A}$ so that $e \notin \mathcal{J}$.

Proof. Recall from [8, Corollary 2.2] that every element of the spectrum of $\mathcal{A}$ can be identified with a norm one linear functional which maps all matrix units onto $\{0,1\}$. In [8, Proposition 3.5] it is shown that for every meet irreducible ideal $\mathcal{J}$ there exists a unique functional $\varphi_{\mathcal{J}}$ in the spectrum such that $\varphi_{\mathcal{J}}$ annihilates $\mathcal{J}$ but does not annihilate $\mathcal{J}^{+}$. The proof follows from that of [8, Theorem 3.7]. There it is shown that if $e \in \mathcal{A}_{i}, i \in \mathbb{N}$, is a matrix unit, then there exists a completely meet irreducible ideal $\mathcal{J}$ so that $\varphi_{\mathcal{J}}(e)=1$. Since $\varphi_{\mathcal{J}}$ annihilates $\mathcal{J}$, the conclusion follows.

The following result depends essentially on an application of Arveson's distance formula.

Lemma $2.9([3])$. Let $\mathcal{A}=\underline{\lim }\left(\mathcal{A}_{i}, \varphi_{i}\right)$ be a strongly maximal TAF algebra and let $\mathcal{J} \subseteq \mathcal{A}$ be a closed ideal. If $a \in \mathcal{A}_{i}, i \in \mathbb{N}$, then

$$
\operatorname{dist}\left(a, \mathcal{A}_{i} \cap \mathcal{J}\right)=\operatorname{dist}(a, \mathcal{J}) .
$$

Proof. In [3, Corollary 2.5] we show that the injection of $\mathcal{A}_{i} /\left(\mathcal{A}_{i} \cap \mathcal{J}\right)$ into $\mathcal{A}_{i+1} /$ $\left(\mathcal{A}_{i+1} \cap \mathcal{J}\right)$ is a complete isometry. Therefore, if $a \in \mathcal{A}_{i}$, then

$$
\operatorname{dist}\left(a, \mathcal{A}_{i} \cap \mathcal{J}\right)=\operatorname{dist}\left(a, \mathcal{A}_{i+k} \cap \mathcal{J}\right),
$$

for all $k \in \mathbb{N}$. The above equality passes to closed unions, and since $\mathcal{J}$ is inductive, the conclusion follows.

Theorem 2.10. Let $\mathcal{A}=\underset{\lim }{\longrightarrow}\left(\mathcal{A}_{i}, \varphi_{i}\right)$ be a strongly maximal TAF algebra. If a $\in \mathcal{A}$, then

$$
\|a\|=\sup \{\|\rho(a)\| \mid \rho \text { is a contractive essential nest representation }\} .
$$

Proof. Let $\mathfrak{A}=\lim \left(\mathfrak{A}_{i}, \varphi_{i}\right)$ be the enveloping $\mathrm{C}^{*}$-algebra of $\mathcal{A}$. First we prove the result in the case where $\mathfrak{A}$ is primitive.

Let $a_{i} \in \mathcal{A}_{i}$ so that $\left\|a-a_{i}\right\| \leq \epsilon$ and so $\left\|a_{i}\right\| \geq\|a\|-\epsilon$. Since $\mathfrak{A}$ is primitive there exists a single summand $\mathcal{A}_{j}^{\left(k_{j}\right)}$ in some $\mathcal{A}_{j}, j>i$, so that all summands of $\mathcal{A}_{i}$ are embedded in $\mathcal{A}_{j}^{\left(k_{j}\right)}$. Let $e$ be the top right matrix unit in $\mathcal{A}_{j}^{\left(k_{j}\right)}$ and let $\mathcal{J} \subseteq \mathcal{A}$ be a completely meet irreducible ideal as in Lemma 2.8 so that $e \notin \mathcal{J}$. 
By construction, none of the matrix units in $\mathcal{A}_{i}$ belong to $\mathcal{J}$ or otherwise $e \in \mathcal{J}$. Therefore, $\mathcal{A}_{i} \cap \mathcal{J}=\{0\}$ and so Lemma 2.9 shows that

$$
\operatorname{dist}\left(a_{i}, \mathcal{J}\right)=\left\|a_{i}\right\| \text {. }
$$

Now consider an isometric nest representation $\hat{\rho}$ of $\mathcal{A} / \mathcal{J}$ [4, Theorem 2.4]. Then the induced representation $\rho: \mathcal{A} \rightarrow \operatorname{Alg} \mathcal{N}$ is a contractive essential nest representation and satisfies

Hence

$$
\left\|\rho\left(a_{i}\right)\right\|=\operatorname{dist}\left(a_{i}, \mathcal{J}\right)=\left\|a_{i}\right\| \geq\|a\|-\epsilon .
$$

$$
\begin{aligned}
\|\rho(a)\| & \geq\left\|\rho\left(a_{i}\right)\right\|-\left\|\rho\left(a-a_{i}\right)\right\| \\
& \geq\|a\|-2 \epsilon .
\end{aligned}
$$

Since $\epsilon$ is arbitrary, the conclusion follows.

In order to prove the result in the general case, note that there exists a primitive ideal $\mathfrak{J}$ of $\mathfrak{A}$ so that $\|a\|=\|a+\mathfrak{J}\|$. The natural map $\pi: \mathfrak{A} \rightarrow \mathfrak{A} / \mathfrak{J}$ sends $\mathcal{A}$ on a strongly maximal TAF algebra with primitive enveloping $\mathrm{C}^{*}$-algebra $\mathfrak{A} / \mathfrak{J}$. From what we proved earlier it follows that there exists a contractive essential nest representation $\hat{\rho}$ of $\hat{\mathcal{A}}$ so that

$$
\|\hat{\rho}(a+\mathfrak{J})\| \geq\|a+\mathfrak{J}\|-\epsilon=\|a\|-\epsilon .
$$

Considering the representation $\hat{\rho} \circ \pi$, the conclusion follows.

\section{Structure For nest Representations WITH COMPACT OPERATORS IN THE RANGE}

In this section we study arbitrary nest representations with compact operators in the range. The central result of the section is the dichotomy of Theorem 3.3 either the kernel of an $\mathbb{N}$-ordered nest representation is a prime ideal or else the range of the representation contains non-zero finite rank operators. We also obtain some approximation results, Theorem 3.7 and Corollary 3.8, which are similar in spirit to those of [9, 14.

We begin with a result that limits the types of nests involved in representations of strongly maximal TAF algebras with non-zero compact operators in the ranges. We need the following.

Lemma 3.1. Let $\mathcal{A}=\lim \left(\mathcal{A}_{i}, \varphi_{i}\right)$ be a strongly maximal TAF algebra and let $\rho: \mathcal{A} \rightarrow \operatorname{Alg} \mathcal{N}$ be a nest representation on a Hilbert space $\mathcal{H}$. Assume that $\rho$ is a *-representation on the diagonal $\mathcal{A} \cap \mathcal{A}^{*}$ of $\mathcal{A}$. If $\rho(\mathcal{A})$ contains non-zero compact operators, then $\rho\left(\mathcal{A} \cap \mathcal{A}^{*}\right)^{\prime \prime}$ has atoms.

Proof. Note that the elements of $\mathcal{A}$ that are mapped to compact operators form a closed ideal in $\mathcal{A}$. Since closed ideals of limit algebras are inductive, there exists an $n \in \mathbb{N}$ and a matrix unit $e \in \mathcal{A}_{n}$ so that $\rho(e)$ is a non-zero compact operator. Now $e$ can be expressed as a sum

$$
e=\sum_{l} e_{l}^{(n+1)}
$$

of matrix units $e_{l}^{(n+1)} \in \mathcal{A}_{n+1}$ whose initial and final spaces are orthogonal. Hence, the operators $\rho\left(e_{l}^{(n+1)}\right)$ act on orthogonal subspaces of $\mathcal{H}$ and have orthogonal ranges. Since their sum equals $\rho(e)$ there exists at least one of them, say $e_{n+1}$, so that $\left\|\rho\left(e_{n+1}\right)\right\|=\|\rho(e)\|$. Arguing as above we now produce a subordinate 
$e_{n+2} \in \mathcal{A}_{n+2}$ of $e_{n+1}$ so that $\left\|\rho\left(e_{n+2}\right)\right\|=\|\rho(e)\|$. Inductively, we define a sequence $\left\{e_{i}\right\}_{i \geq n}, e_{i} \in \mathcal{A}_{i}$, of matrix units each of which subordinates the previous one, so that $\left\|\rho\left(e_{i}\right)\right\|=\|\rho(e)\|$ for all $i$. Equivalently, we produce decreasing sequences $\left\{p_{i}\right\}_{i \geq n},\left\{q_{i}\right\}_{i \geq n}$ of diagonal matrix units, $p_{i}, q_{i} \in \mathcal{A}_{i}, i \geq n$, so that $\left\|\rho\left(p_{i} e q_{i}\right)\right\|=$ $\|\rho(e)\|$. Note that the sequences $\left\{\rho\left(p_{i}\right)\right\}_{i \geq n},\left\{\rho\left(q_{i}\right)\right\}_{i \geq n}$ are decreasing sequences of selfadjoint projections and therefore converge strongly to projections $p$ and $q$, respectively. We will show that the desired atom for $\rho\left(\mathcal{A} \cap \mathcal{A}^{*}\right)^{\prime \prime}$ is the projection $p$.

First we prove that $p$ is a non-zero projection. Indeed, $\left\{\rho\left(p_{i}\right) \rho(e) \rho\left(q_{i}\right)\right\}_{i \geq n}$ converges strongly to $p \rho(e) q$. Since $\rho(e)$ is compact, $\left\{\rho\left(p_{i}\right) \rho(e) \rho\left(q_{i}\right)\right\}_{i \geq n}$ converges to $p \rho(e) q$ in norm. However,

$$
\left\|\rho\left(p_{i}\right) \rho(e) \rho\left(q_{i}\right)\right\|=\left\|\rho\left(e_{i}\right)\right\|=\|\rho(e)\|
$$

for all $i$ and so $\|p \rho(e) q\|=\|\rho(e)\|$, i.e., $p$ is a non-zero projection.

Finally, note that for a fixed $i \in \mathbb{N}, p_{i+k} \mathcal{A}_{i} p_{i+k}=\mathbb{C} p_{i+k}$, for all $k \in \mathbb{N}$. Hence, $p \rho\left(\mathcal{A}_{i}\right) p=\mathbb{C} p$ and so $p \rho(\mathcal{A}) p=\mathbb{C} p$. In particular, $p$ is an atom for $\rho\left(\mathcal{A} \cap \mathcal{A}^{*}\right)^{\prime \prime}$, and the conclusion follows.

Theorem 3.2. Let $\mathcal{A}$ be a strongly maximal TAF algebra and let $\rho: \mathcal{A} \rightarrow \operatorname{Alg} \mathcal{N}$ be a nest representation. If $\rho(\mathcal{A})$ contains non-zero compact operators, then $\mathcal{N}$ is similar to a completely atomic nest. Furthermore, $\rho(\mathcal{A})$ is $w^{*}$-dense in $\operatorname{Alg} \mathcal{N}$.

Proof. Recall that any bounded representation of $\mathcal{A} \cap \mathcal{A}^{*}$ is completely bounded [23, Theorem 8.7], and a completely bounded representation is similar to a completely contractive one [23, Theorem 8.1]. Therefore $\rho$ is similar to a nest representation $\hat{\rho}$ so that $\hat{\rho}$ is a $*$-representation on the diagonal of $\mathcal{A}$. Let $S$ be the invertible operator implementing that similarity, i.e., $\hat{\rho}(A)=S \rho(A) S^{-1}$, for all $A \in \mathcal{A}$. The previous lemma shows that $\hat{\rho}\left(\mathcal{A} \cap \mathcal{A}^{*}\right)^{\prime \prime}$ contains an atom. Proposition 3.5 in 12 shows now that $\hat{\rho}\left(\mathcal{A} \cap \mathcal{A}^{*}\right)^{\prime \prime}$ is a totally atomic von Neumann algebra. By Lemma 2.1, the atoms of $\hat{\rho}\left(\mathcal{A} \cap \mathcal{A}^{*}\right)^{\prime \prime}$ are one dimensional and so $\hat{\rho}\left(\mathcal{A} \cap \mathcal{A}^{*}\right)^{\prime \prime}$ is a masa. Since $S \mathcal{N} S^{-1}$ commutes with $\hat{\rho}\left(\mathcal{A} \cap \mathcal{A}^{*}\right)^{\prime \prime}$, it is a completely atomic nest.

To prove the last sentence, note that since $\hat{\rho}\left(\mathcal{A} \cap \mathcal{A}^{*}\right)^{\prime \prime}$ is a totally atomic masa in $\mathcal{B}(\mathcal{H})$, the $\mathrm{w}^{*}$-closure $\overline{\hat{\rho}(\mathcal{A})} \mathrm{w}^{*}$ of $\hat{\rho}(\mathcal{A})$ contains a masa and satisfies Lat $\overline{\hat{\rho}(\mathcal{A})}{ }^{\mathrm{w}^{*}}=$ $S \mathcal{N} S^{-1}$. Therefore, $\overline{\hat{\rho}}(\mathcal{A})^{\mathrm{w}^{*}}=\operatorname{Alg} S \mathcal{N} S^{-1}$, by [1, Corollary 15.12] and so $\rho(\mathcal{A})$ is $\mathrm{w}^{*}$-dense in $\operatorname{Alg} \mathcal{N}$.

Having established that the nests involved in our considerations are completely atomic (up to a similarity), we now undertake the simplest possible case. Recall that in [4] we proved that the kernel of any nest representation is a meet irreducible ideal. A particular class of meet irreducible ideals, the primitive ideals, were characterized in [3] as the closed and prime ones. The proof of the result below works for any complex algebra, not just strongly maximal TAF algebras. We therefore state it in that generality.

Theorem 3.3. Let $\mathcal{A}$ be a complex algebra and let $\mathcal{N}$ be a maximal nest which is ordered as $\mathbb{N} \cup\{\infty\}$. Let $\rho$ be a representation of $\mathcal{A}$ so that $\rho(\mathcal{A})$ is $w^{*}$-dense in $\operatorname{Alg} \mathcal{N}$. Then the following are equivalent:

(i) $\rho(\mathcal{A})$ contains non-zero finite rank operators.

(ii) $\operatorname{ker} \rho$ fails to be a prime ideal. 
Proof. Assume first that (ii) is valid and by way of contradiction assume that $\rho(\mathcal{A})$ contains no non-zero finite rank operators.

Let $\mathcal{J}_{1}, \mathcal{J}_{2}$ be ideals in $\mathcal{A}$ so that $\mathcal{J}_{1}, \mathcal{J}_{2} \nsubseteq \mathbb{k e r} \rho$. Therefore the closed subspaces $\left[\rho\left(\mathcal{J}_{i}\right)(\mathcal{H})\right], i=1,2$, are non-zero invariant subspaces for $\operatorname{Alg} \mathcal{N}$. Since $\rho(\mathcal{A})$ contains no finite rank operators, any non-zero operator in $\rho\left(\mathcal{J}_{i}\right)$ has infinite-dimensional range and so we necessarily have

$$
\left[\rho\left(\mathcal{J}_{1}\right)(\mathcal{H})\right]=\left[\rho\left(\mathcal{J}_{2}\right)(\mathcal{H})\right]=\mathcal{H} .
$$

Let $a \in \mathcal{J}_{1}$ and $h \in \mathcal{H}$ so that $\rho(a) h \neq 0$. Since $\left[\rho\left(\mathcal{J}_{2}\right)(\mathcal{H})\right]=\mathcal{H}$ there exist sequences $\left\{b_{n}^{(i)}\right\}_{n, i \in \mathbb{N}}$ and $\left\{h_{n}^{(i)}\right\}_{n, i \in \mathbb{N}}$ so that $\lim _{n \rightarrow \infty} \sum_{i} \rho\left(b_{n}^{(i)}\right) h_{n}^{(i)}=h$. Hence,

$$
\lim _{n \rightarrow \infty} \sum_{i} \rho\left(a b_{n}^{(i)}\right) h_{n}^{(i)}=\rho(a) h \neq 0,
$$

and therefore at least one of the terms $\rho\left(a b_{n}^{(i)}\right) h_{n}^{(i)}$ is non-zero. Hence $\mathcal{J}_{1} \mathcal{J}_{2} \not \subset$ ker $\rho$ and so $\operatorname{ker} \rho$ is prime, a contradiction.

We now prove that (i) implies (ii). Assume that $\rho(\mathcal{A})$ contains a non-zero finite rank operator $G$.

Claim 1. $\rho(\mathcal{A})$ contains a non-zero finite rank operator $F$ with zero diagonal.

If $G$ has zero diagonal, then let $F=G$. Otherwise, the finite rank operator $G$ has non-zero eigenvalues $\lambda$. The corresponding Riesz idempotents $E(\lambda), \lambda \in \mathbb{C}$, have finite rank and belong to the algebra generated by $G$ and hence to $\rho(\mathcal{A})$. Choose any such non-zero $E(\lambda)$ and let $S=\left[E(\lambda)^{*} E(\lambda)-(I-E(\lambda))^{*}(I-E(\lambda))\right]^{1 / 2}$ be the normalizer of $E(\lambda)$. Thus $P \doteq S E(\lambda) S^{-1}$ is a selfadjoint projection that belongs to the diagonal of the nest $S \mathcal{N}$. (By a result of Larson $S \mathcal{N}$ is unitarily equivalent to $\mathcal{N}$ and therefore an $\mathbb{N}$-ordered nest.) Note that a similarity preserves the Jacobson Radical and therefore finite rank operators with zero diagonal. Hence it is enough to prove the claim for $S \rho(\mathcal{A}) S^{-1}$. We distinguish two cases.

If $\operatorname{dim} P \geq 2$, then $P S \rho(\mathcal{A}) S^{-1} P$ is w*-dense and therefore equal to $P(\operatorname{Alg} S \mathcal{N}) P$ (Theorem 3.2). Since $P(\operatorname{Alg} S \mathcal{N}) P$ contains rank one operators with zero diagonal, the same is true for

$$
P S \rho(\mathcal{A}) S^{-1} P=S E(\lambda) \rho(\mathcal{A}) E(\lambda) S^{-1} \subset S \rho(\mathcal{A}) S^{-1},
$$

which proves the claim in that case.

If $\operatorname{dim} P=1$, then $P \operatorname{Alg} S \mathcal{N}$ consists of rank one operators and has

$$
P S \rho(\mathcal{A}) S^{-1}=S E(\lambda) \rho(\mathcal{A}) S^{-1}
$$

as a dense subset. Hence there exists at least one non-zero rank one operator in $S E(\lambda) \rho(\mathcal{A}) S^{-1}$, say $R$. The desired finite rank operator will then be any operator of the form

$$
F=R-t P
$$

for a suitable scalar $t$. The proof of the claim is complete.

Let $F$ be as in the Claim 1 and assume that $F$ has rank $n$. By [10, Lemma 1.2], $F$ can be expressed as the sum of $n$ rank one operators in $\operatorname{Alg} \mathcal{N}$ with zero diagonal. Hence there exist projections $E_{i} \in \mathcal{N}, 1 \leq i \leq n$, and vectors $e_{i} \in E_{i}^{\perp}, f_{i} \in E_{i}$, $1 \leq i \leq n$, so that

$$
F=\sum_{i=1}^{n} e_{i} \otimes f_{i} .
$$


Let $x$ be an element of $\mathcal{A}$ so that $\rho(x)=F$ and let $\mathcal{I}_{x}$ be the smallest ideal of $\mathcal{A}$ containing $x$.

Claim 2. $\left(\mathcal{I}_{x}\right)^{n+1} \subseteq \operatorname{ker} \varphi$.

It is enough to show that $\left(\mathcal{I}_{F}\right)^{n+1}=0$, where $\mathcal{I}_{F}$ denotes the smallest ideal of $\operatorname{Alg} \mathcal{N}$ containing $F$. Observe that $\left(\mathcal{I}_{F}\right)^{n+1}$ consists of finite sums of elements of the form

$$
A_{i_{1}}\left(e_{i_{1}} \otimes f_{i_{1}}\right) A_{i_{2}}\left(e_{i_{2}} \otimes f_{i_{2}}\right) \ldots A_{i_{n+1}}\left(e_{i_{n+1}} \otimes f_{i_{n+1}}\right) A_{i_{n+2}}
$$

where $A_{i_{j}} \in \operatorname{Alg} \mathcal{N}$, for all $i_{j}$. At least two of the rank one operators $e_{i_{j}} \otimes f_{i_{j}}$ coincide and therefore the term in (4) reduces to

$$
A\left(e_{i_{j}} \otimes f_{i_{j}}\right) B\left(e_{i_{j}} \otimes f_{i_{j}}\right) C
$$

with $A, B, C \in \operatorname{Alg} \mathcal{N}$. However,

$$
\begin{aligned}
A\left(e_{i_{j}} \otimes f_{i_{j}}\right) B\left(e_{i_{j}} \otimes f_{i_{j}}\right) C & =A\left(E_{i_{j}}^{\perp} e_{i_{j}} \otimes f_{i_{j}}\right) B\left(e_{i_{j}} \otimes E_{i_{j}} f_{i_{j}}\right) C \\
& =A\left(e_{i_{j}} \otimes f_{i_{j}}\right) E_{i_{j}}^{\perp} B E_{i_{j}}\left(e_{i_{j}} \otimes f_{i_{j}}\right) C \\
& =0
\end{aligned}
$$

since $B \in \operatorname{Alg} \mathcal{N}$. This proves the claim.

Let $m$ be the least positive integer so that $\left(\mathcal{I}_{x}\right)^{m} \subseteq \operatorname{ker} \varphi$. Since $\mathcal{I}_{x} \nsubseteq \mathbb{k e r} \rho$, Claim 2 shows that $m$ exists and $m \geq 2$. Consider the ideals $\mathcal{I}=\mathcal{I}_{x}$ and $\mathcal{J}=\left(\mathcal{I}_{x}\right)^{m-1}$. Both are non-trivial ideals not contained in $\operatorname{ker} \rho$ that satisfy $\mathcal{I} \mathcal{J}=\left(\mathcal{I}_{x}\right)^{m} \subseteq \operatorname{ker} \varphi$. Hence ker $\rho$ is not a prime ideal, as desired.

All examples of $\mathbb{N}$-ordered nest representations of TUHF (triangular UHF) algebras available in the literature fail to contain non-zero compact operators in the range. (The known examples of such representations with compact operators in the range occur for TAF algebras whose $\mathrm{C}^{*}$-envelope is not simple.) The following example shows that such representations do occur in a rather natural manner.

Example 3.4. There exists a strongly maximal TUHF algebra $\mathcal{A}=\lim \left(\mathcal{A}_{i}, \varphi_{i}\right)$ which admits an $\mathbb{N}$-ordered nest representation with non-zero rank one operators in the range.

Let $\mathcal{A}_{i}, i \geq 2$, be the collection of all $3^{i} \times 3^{i}$-upper triangular matrices. These are viewed as $3 \times 3$-block upper triangular matrices of the form

$$
\left(\begin{array}{ccc}
A_{1,1} & A_{1,2} & A_{1,3} \\
0 & A_{2,2} & A_{2,3} \\
0 & 0 & A_{3,3}
\end{array}\right),
$$

where both the $(2,2)$ and $(3,3)$ entries are $2^{i} \times 2^{i}$-upper triangular matrices. The embedding $\varphi_{i}$ maps (5) onto the matrix

$$
\left(\begin{array}{ccc}
A_{1,1}^{(3)} & A_{1,2}^{(3)} & A_{1,3}^{(3)} \\
0 & A_{2,2}^{(3)} & A_{2,3}^{(3)} \\
0 & 0 & A_{3,3}^{(3)}
\end{array}\right),
$$

where

$$
A_{k, l}^{(3)}=\left(\begin{array}{ccc}
A_{k, l} & 0 & 0 \\
0 & A_{k, l} & 0 \\
0 & 0 & A_{k, l}
\end{array}\right)
$$


is the threefold ampliation of $A_{k, l}$. (This is a variant of the familiar block-standard embedding.)

Consider the collection $\mathcal{J}_{i}$ of all elements of $\mathcal{A}_{i}$ whose $(2,2)$ and $(3,3)$ entries in (5) are zero. Evidently, $\mathcal{J}_{i} \subseteq \mathcal{A}_{i}$ is an ideal satisfying $\varphi_{i}\left(\mathcal{A}_{i}\right) \cap \mathcal{J}_{i+1}=\varphi_{i}\left(\mathcal{J}_{i}\right)$. Therefore the sequence $\left\{\mathcal{J}_{i}\right\}_{i \in \mathbb{N}}$ determines a closed ideal $\mathcal{J}$ so that $\mathcal{J} \cap \mathcal{A}_{i}=\mathcal{J}_{i}$, $i \in \mathbb{N}$. The quotient $\mathcal{A} / \mathcal{J}$ is the strongly maximal TAF algebra given by the inductive limit

$$
T_{2} \oplus T_{2} \stackrel{\psi_{1}}{\longrightarrow} T_{4} \oplus T_{4} \stackrel{\psi_{2}}{\longrightarrow} T_{8} \oplus T_{8} \stackrel{\psi_{8}}{\longrightarrow} \cdots
$$

so that

$$
\psi_{i}\left(\begin{array}{cc}
A & 0 \\
0 & B
\end{array}\right)=\left(\begin{array}{cc}
\left(\begin{array}{cc}
A & 0 \\
0 & B
\end{array}\right) & 0 \\
0 & \left(\begin{array}{cc}
B & 0 \\
0 & B
\end{array}\right)
\end{array}\right) .
$$

Considering a variant of the Smith representation, one sees that $\mathcal{A} / \mathcal{J}$ admits an $\mathbb{N}$-ordered nest representation whose range contains all upper triangular compact operators, and the conclusion follows.

The reader may have noted that the proof of (i) implies (ii) in Theorem 3.3 is valid for any maximal nest $\mathcal{N}$. Therefore

Corollary 3.5. Let $\mathcal{A}$ be a complex algebra, let $\mathcal{N}$ be a maximal nest and let $\rho$ be a representation of $\mathcal{A}$ so that $\rho(\mathcal{A})$ is $w^{*}$-dense in $\operatorname{Alg} \mathcal{N}$. If $\operatorname{ker} \rho$ is a prime ideal, then $\rho(\mathcal{A})$ does not contain any non-zero finite rank operators.

The refinement algebras are not semisimple, and therefore the trivial ideal $\{0\}$ is not prime in these algebras [3. On the other hand, there exist many representations of the refinement algebras whose ranges do not contain any non-zero compact operators. This shows that the converse of Corollary 3.5 fails for nest representations of order different than $\mathbb{N}$.

Note that in the proof of (i) implies (ii) in Theorem 3.3. it follows that if $\rho(\mathcal{A})$ contains finite rank operators, then $\mathcal{A} / \operatorname{ker} \rho$ cannot be semisimple and so $\operatorname{ker} \rho$ cannot be the intersection of any collection of primitive ideals.

Corollary 3.6. Let $\mathcal{A}$ be a strongly maximal TAF algebra and let $\rho$ be an $\mathbb{N}$-ordered nest representation of $\mathcal{A}$ on a Hilbert space $\mathcal{H}$. Then either $\operatorname{ker} \rho$ is a primitive ideal or ker $\rho$ is a meet irreducible ideal which cannot be expressed as the intersection of primitive ideals. In that case, $\rho(\mathcal{A})$ contains non-zero finite rank operators.

For the rest of this section, we specialize on nest representations with closed range. Such representations exist in abundance. Indeed, Theorem 2.4 in [4] implies that for any meet irreducible ideal $\mathcal{J}$ of a TAF algebra $\mathcal{A}$, there exists a nest representation $\rho: \mathcal{A} \rightarrow \operatorname{Alg} \mathcal{N}$ with closed range so that $\operatorname{ker} \rho=\mathcal{J}$. We begin with an approximation result that has already been used in Example 2.6.

Theorem 3.7. Let $\mathcal{A}=\underline{\lim }\left(\mathcal{A}_{i}, \varphi_{i}\right)$ be a strongly maximal TAF algebra and let $\rho: \mathcal{A} \rightarrow \operatorname{Alg} \mathcal{N}$ be a nest representation with closed range. Let $e \in \bigcup_{i \in \mathbb{N}} \mathcal{A}_{i}$ be an element of $\mathcal{A}$ so that $\rho(e)$ is a compact operator. Then $\rho(e)$ can be expressed as the sum of finitely many rank one operators in $\rho\left(\bigcup_{i \in \mathbb{N}} \mathcal{A}_{i}\right)$.

Proof. Let $\mathcal{J}=\operatorname{ker} \rho$ and let $\hat{\rho}: \mathcal{A} / \mathcal{J} \rightarrow \operatorname{Alg} \mathcal{N}$ be the mapping $\hat{\rho}(a+\mathcal{J})=\rho(a)$, $a \in \mathcal{A}$. Since $\rho(\mathcal{A})$ is closed, the Inverse Mapping Theorem implies that $\hat{\rho}$ is an 
isomorphism of Banach spaces and so there exist non-zero constants $c_{1}$ and $c_{2}$ so that

$$
c_{1}\|a+\mathcal{J}\| \leq\|\rho(a)\| \leq c_{2}\|a+\mathcal{J}\| .
$$

It is enough to prove that for any matrix unit $e \in \mathcal{A}, \rho(e)$ can be expressed as the sum of finitely many rank one operators in $\rho\left(\bigcup_{i \in \mathbb{N}} \mathcal{A}_{i}\right)$. A matrix unit $e \in \mathcal{A}_{n}$ is said to be elementary iff $\rho(e) \neq 0$ and all but one of its subordinates in $\mathcal{A}_{i}, i \geq n$, are annihilated by $\rho$. An argument identical to that in the proof of Theorem 2.3 shows that if $e$ is elementary, then $\rho(e)$ is a rank one operator.

We claim that $e \in \mathcal{A}_{n}$ is a finite sum of elementary operators. Equivalently, we claim that the number of subordinates of $e$ in $\mathcal{A}_{i}$, not annihilated by $\rho$, is uniformly bounded. Clearly the claim proves the theorem.

By way of contradiction, assume that the number of subordinates of $e$ in $\mathcal{A}_{i}$, not annihilated by $\rho$, is not uniformly bounded. Then there exist two subordinates $e_{1}, f_{1} \in A_{i_{1}}$ of $e$ so that $\rho\left(e_{1}\right), \rho\left(f_{1}\right) \neq 0$, and the number subordinates of $e_{1}$ in $\mathcal{A}_{i}, i \geq i_{1}$, not annihilated by $\rho$ is not uniformly bounded. Consequently, there exist two subordinates $e_{2}, f_{2} \in A_{i_{2}}$ of $e_{1}$ so that $\rho\left(e_{2}\right), \rho\left(f_{2}\right) \neq 0$ and the number subordinates of $e_{2}$ in $\mathcal{A}_{i}, i \geq i_{2}$, not annihilated by $\rho$ is, once again, not uniformly bounded. This way we produce a sequence $\left\{f_{i}\right\}_{i \in \mathbb{N}}$ of subordinates of $e$ so that $\rho\left(f_{i}\right) \neq 0$. Furthermore, if $q_{i}$ and $p_{i}$ are the initial and final spaces of $f_{i}, i \in \mathbb{N}$, then

$$
p_{i} p_{j}=q_{i} q_{j}=0
$$

for all $i, j \in \mathbb{N}$, with $i \neq j$.

Since $\rho$ is a ${ }^{*}$-representation on the diagonal, $\left\{\rho\left(p_{i}\right)\right\}_{i \in \mathbb{N}}$ and $\left\{\rho\left(q_{i}\right)\right\}_{i \in \mathbb{N}}$ are sequences of mutually orthogonal projections and therefore converge strongly to 0 . Since $\rho(e)$ is a compact operator we obtain

$$
\lim _{i \in \mathbb{N}}\left\|\rho\left(f_{i}\right)\right\|=\lim _{i \in \mathbb{N}}\left\|\rho\left(p_{i}\right) \rho(f) \rho\left(q_{i}\right)\right\|=0 .
$$

On the other hand,

$$
\operatorname{dist}\left(f_{i}, \mathcal{A}_{i} \cap \mathcal{J}\right)=\operatorname{dist}\left(f_{i}, \mathcal{J}\right)=1,
$$

and so (7) implies that $\left\|\rho\left(f_{i}\right)\right\| \geq c_{1}$, which is a contradiction, and the conclusion follows.

Corollary 3.8. Let $\mathcal{A}=\lim \left(\mathcal{A}_{i}, \varphi_{i}\right)$ be a strongly maximal TAF algebra and let $\rho: \mathcal{A} \rightarrow \operatorname{Alg} \mathcal{N}$ be a nest representation with closed range. Then every compact operator in $\rho(\mathcal{A})$ can be approximated in norm by sums of rank one operators in $\rho(\mathcal{A})$. In particular, $\rho(\mathcal{A})$ contains a non-zero compact operator if and only if it contains a non-zero rank one operator.

Proof. The elements of $\mathcal{A}$ that are mapped to compact operators form a closed ideal $\mathcal{K}$ in $\mathcal{A}$. Since the closed ideals of limit algebras are inductive, any such element $a \in \mathcal{K}$ can be approximated by elements in $\bigcup_{i \in \mathbb{N}}\left(\mathcal{A}_{i} \cap \mathcal{K}\right)$. The previous theorem shows that $\rho\left(\bigcup_{i \in \mathbb{N}}\left(\mathcal{A}_{i} \cap \mathcal{K}\right)\right)$ consists of sums of rank one operators in $\rho(\mathcal{A})$, and the conclusion follows.

Theorem 3.9. Let $\mathcal{A}=\underline{\lim }\left(\mathcal{A}_{i}, \varphi_{i}\right)$ be a strongly maximal TAF algebra and let $\rho: \mathcal{A} \rightarrow \operatorname{Alg} \mathcal{N}$ be a nest representation with closed range. Then the compact operators in $\rho(\mathcal{A})$ form a closed ideal of $\operatorname{Alg} \mathcal{N}$. 
Proof. Corollary 3.8 shows that it is enough to check products between elements of $\operatorname{Alg} \mathcal{N}$ and rank one operators in $\rho(\mathcal{A})$. Let $e \otimes f$ be such a rank one operator and let $A \in \operatorname{Alg} \mathcal{N}$. By Theorem 3.2 there exist nets $\left\{B_{i}\right\}_{i}$ and $\left\{C_{j}^{*}\right\}_{j}, B_{i}, C_{j} \in \rho(\mathcal{A})$, converging to $A$ and $A^{*}$, respectively, in the strong operator topology. Hence, $\left\{B_{i}(e \otimes f)\right\}_{i}$ and $\left\{(e \otimes f) C_{j}\right\}_{j}$ are nets in $\rho(\mathcal{A})$ converging in norm to $A(e \otimes f)$ and $(e \otimes f) A$, respectively. Since $\rho(A)$ is closed, the conclusion follows.

Theorem 3.9 allows us to take advantage of the theory in [10]. For instance, if $\rho(\mathcal{A})$ is closed, then every rank $n$ operator in $\rho(\mathcal{A})$ can be written as the sum of $n$ rank one operators of $\rho(\mathcal{A})([10$, Lemma 1.2]). This improves Corollary 3.8 in that case. We can also improve on Theorems 2.3 and 3.3 as well.

Corollary 3.10. Let $\mathcal{A}=\underline{\lim }\left(\mathcal{A}_{i}, \varphi_{i}\right)$ be a strongly maximal TAF algebra and let $\rho: \mathcal{A} \rightarrow \operatorname{Alg} \mathcal{N}$ be a nest representation with closed range. If $\operatorname{dim} 0_{+}=\operatorname{dim} I_{-}^{\perp}=1$, then the following are equivalent:

(i) $\rho(\mathcal{A})$ contains non-zero compact operators.

(ii) $\operatorname{ker} \rho$ is a completely meet irreducible ideal of $\mathcal{A}$.

Proof. Without loss of generality we may assume that $\rho$ is a $*$-representation on the diagonal. If $\rho(\mathcal{A})$ contains non-zero compact operators, then Theorem 3.9 shows that the compact operators in $\rho(\mathcal{A})$ form a closed ideal of $\operatorname{Alg} \mathcal{N}$. Therefore, $\rho(\mathcal{A})$ contains the rank one operator $R=g \otimes h$ from item (ii) in Theorem 2.3 and so $\operatorname{ker} \rho$ is a completely meet irreducible ideal of $\mathcal{A}$. Conversely, if $\operatorname{ker} \rho$ is a completely meet irreducible ideal of $\mathcal{A}$, then Theorem 2.3 shows that $\rho(\mathcal{A})$ contains a non-zero rank one operator, and the conclusion follows.

Corollary 3.11. Let $\mathcal{A}=\lim \left(\mathcal{A}_{i}, \varphi_{i}\right)$ be a strongly maximal TAF algebra and let $\rho: \mathcal{A} \rightarrow \operatorname{Alg} \mathcal{N}$ be a nest representation with closed range. If $\mathcal{N}$ is a maximal nest which is ordered as $\mathbb{N} \cup\{\infty\}$, then the following are equivalent:

(i) $\rho(\mathcal{A})$ contains non-zero compact operators.

(ii) $\operatorname{ker} \rho$ fails to be a prime ideal of $\mathcal{A}$.

Proof. The result follows from Theorems 3.8 and 3.3 .

\section{SpeCtra AND REPRESENTAtion theory For Limit ALgebras}

In this section we use representation theory to describe an invariant for isometric isomorphisms of separable operator algebras that depends only on the algebra and its diagonal. Subsequently we show that for strongly maximal TAF algebras this invariant coincides with the fundamental relation of Power [26].

Definition 4.1. If $\mathcal{A}$ is an operator algebra, then $\operatorname{nrep}_{*}(\mathcal{A})$ denotes the collection of all nest representations $\rho: \mathcal{A} \rightarrow \mathcal{B}(\mathcal{H})$ of $\mathcal{A}$ so that $\rho$ is a $*$-representation on the diagonal $\mathcal{A} \cap \mathcal{A}^{*}$.

Let $\rho \in \operatorname{nrep}_{*}(\mathcal{A})$ and let $g, h \in \mathcal{H}$ be vectors so that the subspaces $[g],[h]$ are atoms, i.e., minimal intervals, for Lat $\rho(\mathcal{A})$. (Note that if $\mathcal{A}$ is a strongly maximal TAF algebra, the existence of $[g],[h]$ implies that $\rho\left(\mathcal{A} \cap \mathcal{A}^{*}\right)^{\prime \prime}$ is a totally atomic masa and so $\mathcal{N}$ is totally atomic as well.) We define

$$
\omega_{g, h}^{(\rho)}(a)=\langle\rho(a) g, h\rangle, \quad a \in \mathcal{A} .
$$


The collection of all linear forms of the form $\omega_{g, h}^{(\rho)}, \rho \in \operatorname{nrep}_{*}(\mathcal{A}), g, h \in \mathcal{H}$, is denoted as $\Omega_{\mathcal{A}}$. We do not wish to distinguish between multiples of the same form. We therefore define an equivalence relation $\sim$ on $\Omega_{\mathcal{A}}$ to mean

$$
\omega_{g, h}^{(\rho)} \sim \omega_{g^{\prime}, h^{\prime}}^{\left(\rho^{\prime}\right)}
$$

if and only if $\omega_{g, h}^{(\rho)}=\lambda \omega_{g^{\prime}, h^{\prime}}^{\left(\rho^{\prime}\right)}$, for some (non-zero) scalar $\lambda \in \mathbb{C}$. Now consider the quotient space $\Omega_{\mathcal{A}} / \sim$ and define a metric $d$ on $\Omega_{\mathcal{A}} / \sim$ by the formula

$$
d\left(\left[\omega_{1}\right],\left[\omega_{2}\right]\right)=\sum_{n=1}^{\infty} \frac{1}{2^{n}}\left|\frac{\left|\omega_{1}\left(a_{n}\right)\right|}{\left\|\omega_{1}\right\|}-\frac{\left|\omega_{2}\left(a_{n}\right)\right|}{\left\|\omega_{2}\right\|}\right|,
$$

where $\left\{a_{n}\right\}_{n=1}^{\infty}$ is a dense subset of the unit ball of $\mathcal{A}$. A moment's reflection shows that a sequence $\left\{\left[\omega_{k}\right]\right\}_{k=1}^{\infty}$ converges to some $[\omega] \in \Omega_{\mathcal{A}} / \sim$ with respect to the metric $d$ iff

$$
\lim _{k \rightarrow \infty} \frac{\left|\omega_{k}(a)\right|}{\left\|\omega_{k}\right\|}=\frac{|\omega(a)|}{\|\omega\|}
$$

for all $a \in \mathcal{A}$. So even though the definition of the metric $d$ depends on the choice of the subset $\left\{a_{n}\right\}_{n=1}^{\infty}$, the topology $\mathcal{T}$ determined by $d$ is independent of that set.

Definition 4.2. If $\mathcal{A}$ is a separable operator algebra, then the projective dual of $\mathcal{A}$ is the topological space $\left(\Omega_{\mathcal{A}} / \sim, \mathcal{T}\right)$.

The adjective "projective" reflects the similarities between the above construction and the definition of the projective space in algebraic geometry. Since isometric isomorphisms preserve the diagonal, the projective dual is an invariant for isometric isomorphisms.

We now compare the projective dual of a strongly maximal TAF algebra to its fundamental relation. Recall from [8] that the spectrum or fundamental relation of a limit algebra $\mathcal{A}=\underline{\lim }\left(\mathcal{A}_{i}, \varphi_{i}\right)$ is the set

$$
\operatorname{Spec}(\mathcal{A})=\left\{\omega \in \mathcal{A}^{\#} \mid\|\omega\|=1 \text { and } \omega\left(\bigcup_{i=1}^{\infty} \mathcal{C}_{i}\right) \subseteq\{0,1\}\right\}
$$

equipped with the relative $\mathrm{w}^{*}$-topology as a subset of the dual $\mathcal{A}^{\#}$ of the Banach space $\mathcal{A}$. Here $\mathcal{C}_{i}$ denotes the natural matrix unit system associated with $\mathcal{A}_{i}$. (The spectrum of $\mathcal{A}$ is also equipped with a partially defined operation that makes it a topological semigroupoid; we will come to these details later.)

Lemma 4.3. If $\mathcal{A}=\underline{\lim }\left(\mathcal{A}_{i}, \varphi_{i}\right)$ is a strongly maximal TAF algebra, then $\operatorname{Spec}(\mathcal{A})$ $\subseteq \Omega_{\mathcal{A}}$. Conversely, if $\vec{\omega} \in \Omega_{\mathcal{A}}$, then there exists a non-zero scalar $\lambda$ so that $\lambda \omega \in$ $\operatorname{Spec}(\mathcal{A})$.

Proof. Let $\omega \in \operatorname{Spec}(\mathcal{A})$. There exist decreasing sequences $\left\{p_{i}\right\}_{i=1}^{\infty}$ and $\left\{q_{i}\right\}_{i=1}^{\infty}$, $p_{i}, q_{i} \in \mathcal{A}_{i}, i \in \mathbb{N}$, of diagonal projections and a matrix unit $e \in \mathcal{A}_{n}$ so that

$$
\omega\left(q_{i} e p_{i}\right)=1
$$

and $\omega(f)=0$ for all other matrix units $f \in \mathcal{A}_{i}, i \geq n$. The sequence $\left\{q_{i} e p_{i}\right\}_{i=1}^{\infty}$ of subordinates of $e$ determines a path $\Gamma$ on the Bratelli diagram for the enveloping $\mathrm{C}^{*}$-algebra $\mathfrak{A}=\underline{\lim }\left(\mathfrak{A}_{i}, \varphi_{i}\right)$ of $\mathcal{A}$. Let $\mathfrak{J}$ be the ideal consisting of all summands of $\mathfrak{A}=\underline{\lim }\left(\mathfrak{A}_{i}, \overrightarrow{\left.\varphi_{i}\right)}\right.$ that are never mapped into $\Gamma$ and let $\pi: \mathfrak{A} \rightarrow \mathfrak{A} / \mathfrak{J}$ be the quotient map. Then $\pi(\mathcal{A})$ is a strongly maximal TAF algebra of a primitive $\mathrm{C}^{*}$ algebra. Furthermore, the decreasing sequence $\left\{\pi\left(p_{i}\right)\right\}_{i=1}^{\infty}$ of diagonal projections 
determines a multiplicative linear form $p$ on $\pi(\mathcal{A}) \cap \pi(\mathcal{A})^{*}$ whose orbit under the action of $\mathfrak{A} / \mathfrak{J}$ is dense in the Gelfand spectrum of $\pi(\mathcal{A}) \cap \pi(\mathcal{A})^{*}$. We now apply the construction of [22, Proposition II.2.2] so that the role of $\left[x_{0}\right]$ in that proof is played by our $p$. Therefore we obtain a faithful nest representation $\hat{\rho}$ of $\pi(\mathcal{A})$ so that $\hat{\rho}$ is a ${ }^{*}$-representation on the diagonal and Lat $\hat{\rho}(\pi(\mathcal{A}))$ is a maximal, totally atomic nest. In addition, the construction of [22, Proposition II.2.2] implies that the sequences the sequences $\left\{\hat{\rho}\left(\pi\left(p_{i}\right)\right)\right\}_{i=1}^{\infty}$ and $\left\{\hat{\rho}\left(\pi\left(q_{i}\right)\right)\right\}_{i=1}^{\infty}$ converge to onedimensional projections with ranges $[g]$ and $[h]$, respectively. If $\rho=\hat{\rho} \circ \pi$, then it is easy to see that $\omega=\omega_{g, h}^{(\rho)}$, and the conclusion follows.

Conversely, let $\omega \in \Omega_{\mathcal{A}}$ and let $\rho \in \operatorname{nrep}_{*}(\mathcal{A}), g, h \in \mathcal{H}$ so that $\omega=\omega_{g, h}^{(\rho)}$. There exists a matrix unit $e \in \mathcal{A}_{n}$ so that $\omega(e) \neq 0$, and so let $\lambda$ be a scalar so that $\omega(e)=\lambda^{-1}$. Since $\rho$ is a ${ }^{*}$-representation on the diagonal and $[g],[h]$ are atoms for $\rho\left(\mathcal{A} \cap \mathcal{A}^{*}\right)^{\prime \prime}, \omega$ annihilates all but one of the matrix units of $\mathcal{A}_{i}, i \geq n$; let us denote as $e_{i}$ that matrix unit in $\mathcal{A}_{i}$. A moment's reflection shows that $e_{i}$ is a subordinate of $e$ and so $\omega\left(e_{i}\right)=\lambda^{-1}$. It is now clear that $\lambda \omega$ satisfies the requirements of (8), and the proof follows.

Note that Lemma 4.3 establishes the existence of a 1-1 and onto map $\Phi$ : $\left(\operatorname{Spec}(\mathcal{A}), \mathrm{w}^{*}\right) \rightarrow\left(\Omega_{\mathcal{A}} / \sim, \mathcal{T}\right)$. Furthermore, this map is easily seen to be bicontinuous. Hence

Corollary 4.4. The topological spaces $\left(\operatorname{Spec}(\mathcal{A}), w^{*}\right)$ and $\left(\Omega_{\mathcal{A}} / \sim, \mathcal{T}\right)$ are homeomorphic.

In the case of a strongly maximal TAF algebra generated by its order preserving normalizer, Theorem 2.3 shows that the essential nest representations are sufficient to describe the fundamental relation. In the general case one obtains a dense subset of $\left(\Omega_{\mathcal{A}} / \sim, \mathcal{T}\right)$, as $[8$, Theorem 3.7] shows.

The fundamental relation is also equipped with a partially defined operation that makes it a topological semigroupoid. We now indicate how this operation materializes in our representation.

Let $\mathcal{A}$ be a separable operator algebra and let $\omega_{g, h}^{(\rho)}, \omega_{g^{\prime}, h^{\prime}}^{\left(\rho^{\prime}\right)} \in \Omega_{\mathcal{A}}$. If $\rho=\rho^{\prime}$ and $[g]=\left[h^{\prime}\right]$, then we define

$$
\omega_{g, h}^{(\rho)} \circ \omega_{g^{\prime}, h^{\prime}}^{\left(\rho^{\prime}\right)}=\omega_{g^{\prime}, h}^{(\rho)} .
$$

It is not clear at all that (9) establishes a well-defined operation on $\Omega_{\mathcal{A}}$. Nevertheless, this is the case for strongly maximal TAF algebras, as the following result shows.

Theorem 4.5. Let $\mathcal{A}$ be a strongly maximal TAF algebra. Then the triple $\left(\Omega_{\mathcal{A}} / \sim\right.$, $\mathcal{T}, \circ)$ forms a topological semigroupoid which is isomorphic to the fundamental relation of $\mathcal{A}$.

Proof. If $\omega_{g, h}^{(\rho)} \in \Phi(\omega)$, then the last paragraph in the proof of Lemma 4.3 shows that $\omega_{g, g}^{(\rho)}=s(\omega)$ and $\omega_{h, h}^{(\rho)}=r(\omega)$, where $s(\cdot)$ and $r(\cdot)$ are the source and range maps [8]. Hence, if $\omega_{g, h}^{(\rho)} \in \Phi(\omega)$ and $\omega_{g^{\prime}, h^{\prime}}^{\left(\rho^{\prime}\right)} \in \Phi\left(\omega^{\prime}\right)$ are composable, then $r\left(\omega^{\prime}\right)=s(\omega)$ and so $\omega$ and $\omega^{\prime}$ are composable. In addition, $\omega_{g^{\prime}, h}^{(\rho)} \in \Phi\left(\omega \circ \omega^{\prime}\right)$. This shows that $\circ$ is a well-defined operation on $\Omega_{\mathcal{A}} / \sim$ and that $\Phi^{-1}$ respects that operation. 
We now show that $\Phi$ also respects the composition on $\operatorname{Spec}(\mathcal{A})$. Let $\omega, \omega^{\prime} \in$ $\operatorname{Spec}(\mathcal{A})$ with $r\left(\omega^{\prime}\right)=s(\omega)$. Then $r(\omega), s(\omega), r\left(\omega^{\prime}\right)$ and $s\left(\omega^{\prime}\right)$ belong to the same orbit under the action of $\mathcal{A}$ on the Gelfand space of the diagonal. Therefore the construction of [22, Proposition II.2.2] produces a common representation $\rho$ for both $\omega$ and $\omega^{\prime}$ so that $\omega_{g, h}^{(\rho)} \in \Phi(\omega)$ and $\omega_{g^{\prime}, h^{\prime}}^{\left(\rho^{\prime}\right)} \in \Phi\left(\omega^{\prime}\right)$. Since $r\left(\omega^{\prime}\right)=s(\omega)$, the construction of [22, Proposition II.2.2] also guarantees that $g=h^{\prime}$. Hence $\omega_{g, h}^{(\rho)}$ and $\omega_{g^{\prime}, h^{\prime}}^{\left(\rho^{\prime}\right)}$ are composable. In addition,

$$
\Phi\left(\omega \circ \omega^{\prime}\right)=\left[\omega_{g^{\prime}, h}^{\left(\rho^{\prime}\right)}\right]=\left[\omega_{g, h}^{(\rho)}\right] \circ\left[\omega_{g^{\prime}, h^{\prime}}^{\left(\rho^{\prime}\right)}\right]=\Phi(\omega) \circ \Phi\left(\omega^{\prime}\right),
$$

and so $\Phi$ also respects the composition. From this it is easy to see that the operation $\circ$ on $\Omega_{\mathcal{A}} / \sim$ is associative. The bicontinuity of $\Phi$ has been established earlier and the continuity of $\circ$ is easy to prove.

Note that Theorem 4.5 gives yet another proof of the invariance of the fundamental relation under isometric isomorphisms. The triple $\left(\Omega_{\mathcal{A}} / \sim, \mathcal{T}, \circ\right)$ can be calculated for other operator algebras as well, including various semicrossed products. It seems that in the case where $\mathcal{A}$ is the algebra of an r-discrete, principal semigroupoid $\mathcal{G}$ [21, one should be able to relate the triple $\left(\Omega_{\mathcal{A}} / \sim, \mathcal{T}, \circ\right)$ to the semigroupoid $\mathcal{G}$. We plan to pursue these directions elsewhere.

\section{REFERENCES}

[1] K. Davidson, Nest Algebras, Pitman Research Notes in Mathematics Series, 191, 1988. MR0972978 (90f:47062)

[2] K. Davidson, $\mathrm{C}^{*}$-algebras by example, Fields Institute Monographs, American Mathematical Society, 1996. MR.1402012 (97i:46095)

[3] K. Davidson and E. Katsoulis, Primitive limit algebras and $\mathrm{C}^{*}$-envelopes, Adv. Math. 170, 2002, 181-205. MR.1932328 (2003h:47140)

[4] K. Davidson, E. Katsoulis and J. Peters, Meet irreducible ideals and representations of limit algebras, J. Funct. Anal. 200, 2003, 23-30. MR1974086 (2004b:47126)

[5] A. Donsig, A. Hopenwasser, T. Hudson, M. Lamoureux and B. Solel, Meet irreducible ideals in direct limit algebras, Math. Scand. 87, 2000, 27-63. MR1776964 (2001f:47112)

[6] A. Donsig and T. Hudson, The lattice of ideals of a triangular AF algebra, J. Funct. Anal. 138, 1996, 1-39. MR1391629 (97e:47068)

[7] A. Donsig, T. Hudson and E. Katsoulis, Algebraic isomorphisms of limit algebras, Trans. Amer. Math. Soc. 353, 2001, 1169-1182. MR1804417 (2001k:47103)

[8] A. Donsig, D. Pitts, S. Power, Algebraic isomorphisms and spectra of triangular limit algebras, Indiana Univ. Math. J. 50, 2001, 1131-1147. MR1871350 (2002k:47148)

[9] J. Erdos, Operators of finite rank in nest algebras, J. London Math. Soc. 43, 1968, 391-397. MR0230156 (37:5721)

[10] J. Erdos and S. Power, Weakly closed ideals of nest algebras, J. Operator Theory 7, 1982, 219-235. MR0658610(84a:47056)

[11] J. Glimm, Type I C*-algebras, Ann. Math. 73, 1961, 572-612. MR0124756 (23:A2066)

[12] A. Hopenwasser, J. Peters, S. Power, Nest representations of TAF algebras, Canad. J. Math. 52, 2000, 1221-1234. MR1794303 (2001h:47121)

[13] E. Katsoulis and D. Kribs, Isomorphisms of algebras associated with directed graphs, Math. Ann. 330, 2004, 709-728. MR2102309 (2005i:47114)

[14] E. Katsoulis and R. Moore, On compact operators in certain reflexive operator algebras, J. Operator Theory 25, 1991, 177-182. MR1191259 (93i:47065)

[15] D.W. Kribs, S.C. Power, Free semigroupoid algebras, J. Ramanujan Math. Soc. 19, (2004), 117-159. MR2076898 (2005c:47106)

[16] M. Lamoureux, Nest representations and dynamical systems, J. Funct. Anal. 114, 1993, 345-376. MR1223711 (95e:46078) 
[17] M. Lamoureux, Ideals in some continuous nonselfadjoint crossed product algebras, J. Funct. Anal. 142, 1996, 211-248. MR.1419421 (97m:47060)

[18] M. Lamoureux, Some triangular AF algebras, J. Operator Theory 37, 1997, 91-109. MR:1438202 (2000f:47108)

[19] D. Larson and B. Solel, Structured triangular limit algebras, Proc. London Math. Soc. 75, 1997, 177-193. MR1444318 (98c:46153)

[20] P.S. Muhly, A finite dimensional introduction to operator algebra, A. Katavolos (ed.), Operator Algebras and Application, Kluwer Academic Publishers, 1997, 313-354. MR1462686 (98h:46062)

[21] P. Muhly and B. Solel, Subalgebras of groupoid $C^{*}$-algebras, J. Reine Angew. Math. 402, 1989, 41-75. MR 1022793 (90m:46098)

[22] J. Orr and J. Peters, Some representations of TAF algebras, Pacific. J. Math. 167, 1995, 129-161. MR1318167 (96c:46055)

[23] V. I. Paulsen, Completely Bounded Maps and Dilations, Longman Scientific, New York, Wiley, 1986. MR0868472 (88h:46111)

[24] G. K. Pedersen, $C^{*}$-Algebras and their automorphism groups, London Mathematical Society Monograph, Academic Press, 1979. MR0548006 (81e:46037)

[25] J. Peters, Y.T. Poon and B. Wagner, Triangular AF algebras, J. Operator Theory 23, 1990, 81-114. MR 1054818(91h:46102)

[26] S. Power, Classification of tensor products of triangular operator algebras, Proc. London Math. Soc. 61, 1990, 571-614. MR1069516 (92a:47053)

[27] J. R. Ringrose, On some algebras of operators, Proc. London Math. Soc. 15, 1965, 61-83. MR0171174 (30:1405)

[28] Ş. Strătilă and D.V. Voiculescu, Representations of AF-algebras and of the group $U(\infty)$, Springer Lect. Notes Math. 486, Springer-Verlag, Berlin, New York, 1975. MR 0458188 $(56: 16391)$

[29] M. Thelwall, Dilation theory for subalgebras of AF algebras, J. Operator Theory 25, 1991, 275-282. MR1203033 (94a:46079)

Department of Mathematics, East Carolina University, Greenville, North Carolina 27858

E-mail address: katsoulise@ecu.edu

Department of Mathematics, Iowa State University, Ames, Iowa 50011

E-mail address: peters@iastate.edu 